\title{
Impact of Letermovir Use for Cytomegalovirus Prophylaxis on Re-Hospitalization Following Allogeneic Hematopoietic Stem Cell Transplantation: An Analysis of a Phase III Randomized Clinical Trial
}

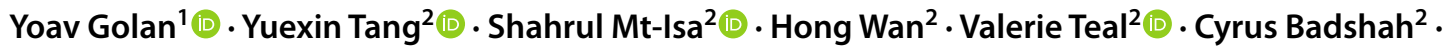 \\ Sanjeet Dadwal ${ }^{3}$
}

Accepted: 17 March 2021 / Published online: 19 April 2021

(c) The Author(s) 2021

\begin{abstract}
Background Allogeneic hematopoietic stem cell transplantation (HSCT) is associated with substantial healthcare resource use, particularly when recipients develop cytomegalovirus (CMV) infection. Letermovir reduced postHSCT CMV infection risk compared with placebo in a previous phase III trial. This analysis evaluated letermovir's impact on re-hospitalization post-transplant.

Methods Using data from a phase III, multicenter, randomized clinical trial (NCT02137772, registered May 14, 2014), this study assessed CMV-associated and all-cause re-hospitalizations at weeks 14, 24, and 48 post-transplant among recipients of letermovir versus placebo. Unstandardized re-hospitalization rates and days were reported; standardized rates and days were estimated accounting for censoring due to death or early study discontinuation.

Results Unstandardized rates (95\% confidence interval [CI]) of all-cause re-hospitalization in letermovir versus placebo recipients at weeks 14,24 , and 48 were $36.6 \%$ (31.4-42.1) versus $47.6 \%$ (39.9-55.4), 49.2\% (43.7-54.8) versus 55.9\% (48.1-63.5), and 55.7\% (50.1-61.2) versus 60.6\% (52.8-68.0), respectively. Unstandardized mean total duration (95\% CI) of re-hospitalization with letermovir versus placebo at weeks 14, 24, and 48 were 7.6 (5.9-9.8) versus 11.3 (8.6-14.8), 13.9 (11.2-17.2) versus 15.5 (11.9-20.1), and 18.0 (14.8-21.9) versus 20.7 (15.8-27.1) days, respectively. Similar results were found in CMV-associated re-hospitalization outcomes and standardized rates and days of all-cause re-hospitalizations.

Conclusions In this post-hoc analysis, letermovir was associated with lower rates of CMV-associated and all-cause rehospitalizations with a shorter length of stay (especially within the first 14 weeks post-transplant).
\end{abstract}

\section{Introduction}

Allogeneic hematopoietic stem cell transplantation (HSCT) is a resource-intensive procedure associated with substantial direct and indirect healthcare costs [1]. HSCT recipients who develop cytomegalovirus (CMV) infection have significantly higher healthcare costs than those without this infection [2,3]; direct hospitalization costs

Sanjeet Dadwal

sdadwal@coh.org

1 Department of Internal Medicine, Division of Infectious Diseases, Tufts Medical Center, Boston, MA, USA

2 Merck \& Co., Inc., Kenilworth, NJ, USA

3 Division of Infectious Diseases, City of Hope National Medical Center, 1500 East Duarte Road, Duarte, CA 91010, USA

\section{Key Points for Decision Makers}

Cytomegalovirus infection in allogeneic hematopoietic stem cell transplantation (HSCT) recipients represents a costly healthcare burden.

This analysis used data from the phase III randomized trial of letermovir versus placebo.

Letermovir was linked to lower rates and fewer days of re-hospitalization post-HSCT.

have been reported to be almost 10-fold higher for HSCT recipients with CMV infection versus those with graftversus-host disease and no CMV infection [4].

Due to the side effects associated with ganciclovir, valganciclovir, and foscarnet, it has been proposed that new 
prophylactic strategies to prevent CMV infection and disease would reduce the burden of this complication, and may ultimately help to improve the outcomes and reduce the cost of allogeneic HSCT [5]. Letermovir is approved by the United States (US) Food and Drug Administration, and is indicated for the prevention of CMV infection and disease in adult CMV-seropositive allogeneic HSCT recipients [6]. In a phase III, double-blind, randomized trial, letermovir significantly reduced the risk of clinically significant CMV infection (CS-CMVi; defined as initiation of pre-emptive therapy for documented viremia or treatment of CMV disease) compared with placebo in adult CMV-seropositive allogeneic HSCT recipients through week 24 post-transplant [7]. In a post-hoc analysis, letermovir reduced all-cause mortality compared with placebo, possibly by preventing or delaying CS-CMVi in recipients of HSCT [8].

This analysis aimed to assess the impact of letermovir use as CMV prophylaxis on rates and duration of re-hospitalization in adult CMV-seropositive allogeneic HSCT recipients from the letermovir phase III clinical trial.

\section{Methods}

The analyses presented here are based on data from a phase III, multicenter, double-blind, placebo-controlled, randomized trial of letermovir for the prevention of CSCMVi in adult recipients of an allogeneic HSCT (ClinicalTrials.gov identifier, NCT02137772). Complete details of the trial design, study population, and primary outcomes have been previously published [7]. Briefly, participants were randomized $2: 1$ to receive letermovir (480 mg/day, adjusted to $240 \mathrm{mg}$ /day with concomitant cyclosporine) or placebo within 28 days post-HSCT through week 14 postHSCT with follow-up through week 48 post-HSCT. The primary endpoint of the study was the proportion of participants with CS-CMVi through week 24 post-HSCT [7]. The population of the Full Analysis Set was used as the primary efficacy study population for these analyses and consisted of all randomized participants who received at least one dose of study medication and had no detectable CMV DNA on day 1.

The outcome of this analysis was re-hospitalization post-transplant. Each re-hospitalization was prospectively recorded via case report form at each site following discharge from the initial hospitalization for HSCT. Participants were followed-up from the time of transplant until death, discontinuation from study, or last visit, whichever was earlier. All-cause re-hospitalization was defined as hospital readmission due to any reason following initial hospital discharge after allogeneic HSCT.
A pre-specified analysis of the phase III trial data was conducted to determine re-hospitalization rates through weeks 14,24 , and 48 post-HSCT. Unstandardized all-cause re-hospitalization rates were calculated as the proportion of participants who had at least one re-hospitalization in each treatment group, and reported with $95 \%$ confidence intervals (CIs) using Clopper-Pearson's exact method. Post hoc analyses included standardized all-cause re-hospitalization rates, and duration of all-cause re-hospitalization through weeks 14, 24, and 48 post-HSCT (unstandardized and standardized). Standardized rates and duration were estimated accounting for censoring due to death or early study discontinuation and reported as rates and days per 100 participant-months. A sensitivity analysis was also performed to account for the number of re-hospitalizations per participant when assessing standardized rates of re-hospitalization; i.e. all re-hospitalization events for a participant are included in the analysis.

A pre-specified analysis of unstandardized CMV-associated re-hospitalization rates was also reported in each treatment group to explore the impact of letermovir on rehospitalizations with CMV infection.

\section{Results}

A total of 570 participants were randomized, of whom 495 participants were included in this analysis where 325 received letermovir and 170 received placebo [7]. Baseline demographics and disease characteristics were similar across the two groups and have been previously reported [7].

Unstandardized rates of all-cause re-hospitalization in letermovir versus placebo recipients at weeks 14,24 , and 48 were $36.6 \%$ versus $47.6 \%$ (95\% CI 31.4-42.1 vs 39.9-55.4), $49.2 \%$ versus $55.9 \%$ (95\% CI $43.7-54.8$ vs $48.1-63.5$ ), and $55.7 \%$ versus $60.6 \%$ (95\% CI $50.1-61.2$ vs $52.8-68.0$ ), respectively (Fig. 1A). Unstandardized CMV-associated re-hospitalization rates were also lower in participants in the letermovir group compared with the placebo group at all three timepoints post-HSCT (Online Resource 1, see electronic supplementary material [ESM]). The standardized all-cause re-hospitalization rates per 100 participantmonths were lower among participants who received letermovir versus placebo at weeks 14,24 , and $48: 12.7$ versus 19.6 (95\% CI $10.9-14.7$ vs $16.3-23.5)$; 9.5 versus 11.1 (95\% CI 8.5-10.7 vs 9.6-12.9); 6.3 versus 7.2 (95\% CI 5.6-7.0 vs 6.2-8.4), respectively (Fig. 1B). A sensitivity analysis accounting for the number of re-hospitalizations also showed consistently lower standardized rates of all-cause re-hospitalization in participants who received letermovir versus placebo (Online Resource 2, see ESM).

Unstandardized mean total duration of re-hospitalization in letermovir versus placebo recipients at weeks 14,24 , and 
Fig. 1 A Unstandardized rates of all-cause re-hospitalization through week 48 post-HSCT. B Standardized rates of all-cause re-hospitalization through week 48 post-HSCT, per 100 participant-months. C Unstandardized mean total duration (days) of all-cause re-hospitalization following HSCT. D Standardized mean total duration (days) of all-cause re-hospitalization following HSCT, per 100 participant-months. 95\% CIs displayed on each graph. ${ }^{\text {a Rates }}$ per 100 participant-months, accounting for early discontinuation or death. ${ }^{\mathrm{b}}$ Total duration (days) per participant for all re-hospitalizations. ${ }^{\text {TTotal dura- }}$ tion (days) per participant for all re-hospitalizations per 100 participant-months, accounting for early discontinuation or death. $C I$ confidence interval, HSCT hematopoietic stem cell transplant
A

Letermovir a Placebo

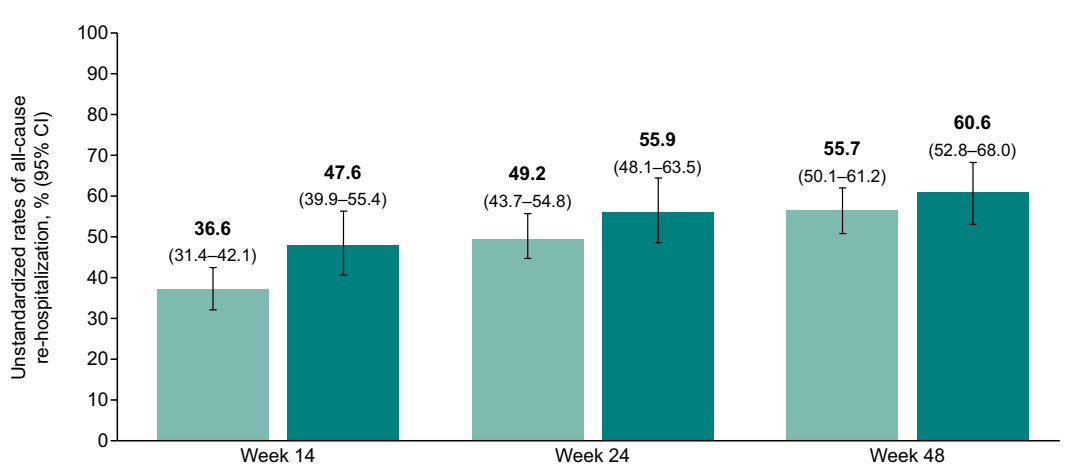

B

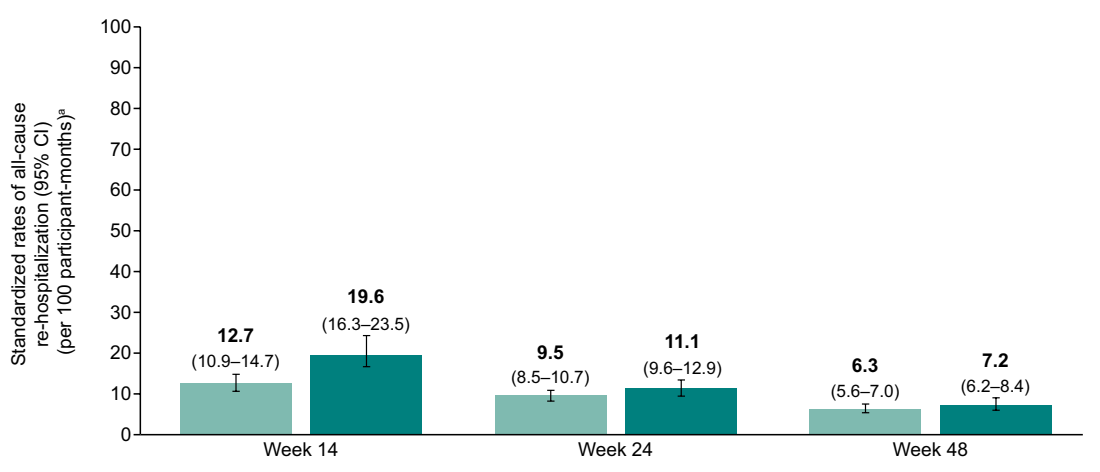

C

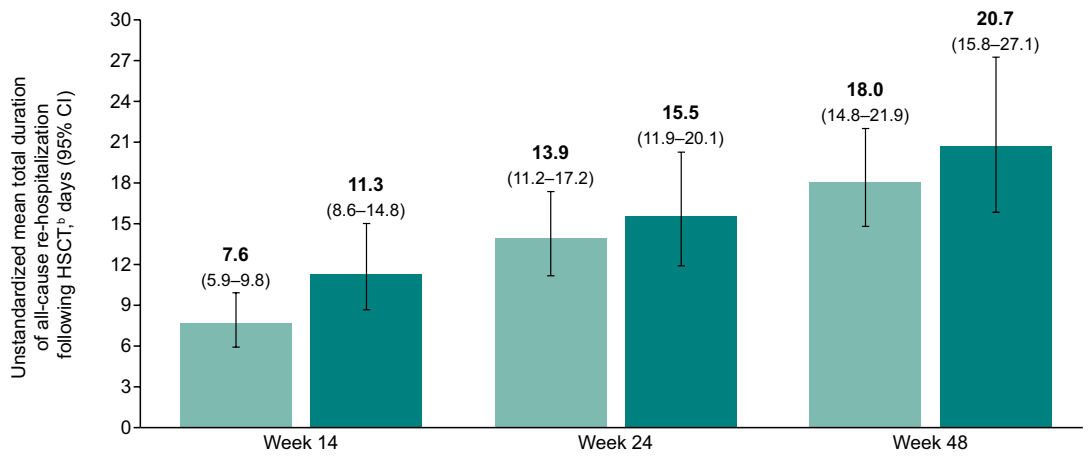

D

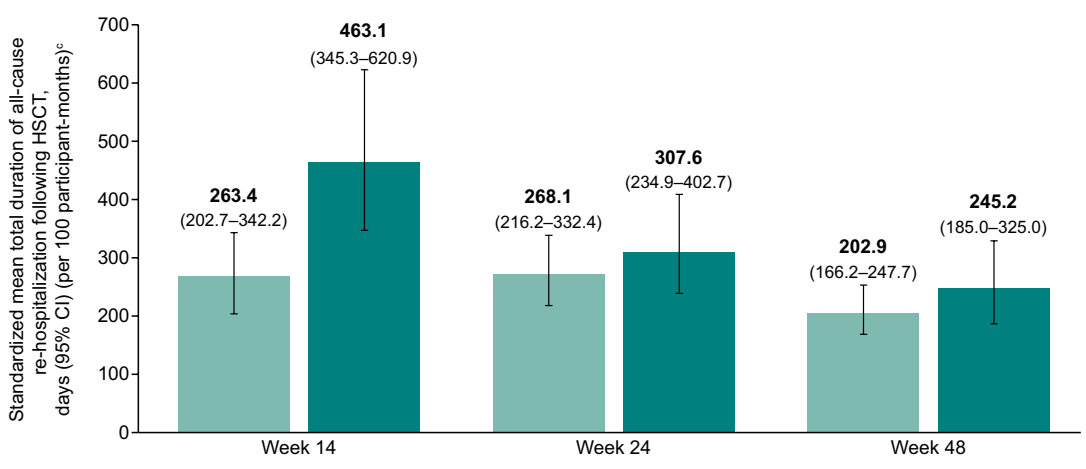


48 were 7.6 versus 11.3 days (95\% CI 5.9-9.8 vs 8.6-14.8), 13.9 versus 15.5 (95\% CI $11.2-17.2$ vs $11.9-20.1)$, and 18.0 versus 20.7 (95\% CI $14.8-21.9$ vs $15.8-27.1$ ), respectively (Fig. 1C). Standardized mean total durations (days) of all-cause re-hospitalization per 100 participant-months in letermovir versus placebo recipients at weeks 14, 24, and 48 were 263.4 versus 463.1 (95\% CI $202.7-342.2$ vs $345.3-620.9$ ), 268.1 versus 307.6 (95\% CI $216.2-332.4$ vs 234.9-402.7), and 202.9 versus 245.2 (95\% CI 166.2-247.7 vs 185.0-325.0), respectively (Fig. 1D).

\section{Discussion}

Anti-CMV prophylaxis with letermovir was associated with a lower rate of re-hospitalizations and shorter length of stay compared with placebo in adult CMV-seropositive allogeneic HSCT recipients, and this effect was more pronounced at 14 weeks post-HSCT. The treatment duration of letermovir was up to 14 weeks post-HSCT, which correlates with the period of highest risk of developing CMV infection [9]. The lower incidence of CS-CMVi in the letermovir group compared with the placebo group through week 24 postHSCT [7] may have been attributable to a lower incidence of re-hospitalizations associated with CMV in participants who received letermovir versus placebo at all timepoints.

Allogeneic HSCT recipients with CMV-associated rehospitalizations within 100 days (approximately 14 weeks) post-HSCT have significantly higher healthcare costs and mortality than those without CMV-associated hospitalization in the US [10]. Even with the use of pre-emptive therapy with valganciclovir, ganciclovir, and foscarnet, CMV infection remains a significant problem after 100 days postHSCT. Indeed, any level of CMV viremia is also associated with increased all-cause mortality [11]. The occurrence of CMV episodes (infection with or without disease) in the first year post-HSCT has been shown to increase the costs of allogeneic HSCT by 25-30\% [5]. By reducing the risk of CSCMVi (thereby decreasing the use of pre-emptive therapy) and re-hospitalization, letermovir may help to reduce the healthcare resource use associated with allogeneic HSCT. A recent health economic model based on this phase III trial and local cost data showed that CMV prophylaxis with letermovir was considered a cost-effective option in adult CMV-seropositive recipients of an allogeneic HSCT from a US payer perspective [12]. These findings may be of particular relevance to US healthcare providers considering the timeframe associated with the bundled payment system used by most US commercial payers [13].

Loss of follow-up due to death or early study discontinuation may lead to a shorter time to observe any healthcare resource use. After adjusting for death and early study discontinuation in the standardized analyses, this study showed letermovir was associated with lower rates and shorter length of all-cause re-hospitalizations post-transplant, compared with placebo. This finding was consistent with the unstandardized analysis.

The following limitations of this study should be considered when interpreting these data. As re-hospitalization was an exploratory endpoint in the phase III trial, it was not powered to detect statistical differences. Due to the complexity of the patient population, it was also difficult to assess reasons for re-hospitalizations. This study does not have information on patient clinical conditions recorded in the re-hospitalization case report form other than whether it was associated with CMV. Healthcare costs were not collected in this global phase III trial, which likely vary substantially across health and reimbursement systems worldwide. Therefore, further health economic modeling and cost-effectiveness analysis are needed to assess the impact of letermovir for CMV prophylaxis from a third-party payer perspective while incorporating country-specific cost data. Finally, the initial hospitalization for transplantation was not included in these analyses. Therefore, the impact of letermovir on the duration of initial hospitalization is unknown. Previous studies showed CMV infection is associated with significantly higher healthcare resource use and cost and letermovir reduced clinically significant CMV infection. This post-hoc analysis may have underestimated the impact of letermovir for CMV prophylaxis on healthcare resource use, as only re-hospitalization data were available in the trial.

\section{Conclusion}

Letermovir prophylaxis reduced rates of re-hospitalization and shortened lengths of stay compared with placebo treatment in CMV-seropositive HSCT recipients. Further research could examine the broader healthcare (i.e., outside of the inpatient setting) and societal impact of letermovir prophylaxis in different healthcare systems in a real-world setting.

Supplementary Information The online version contains supplementary material available at https://doi.org/10.1007/s41669-021-00264-9.

Acknowledgements Medical writing support, under the direction of the authors, was provided by Paul O'Neill, PhD, of CMC AFFINITY, McCann Health Medical Communications, in accordance with Good Publication Practice (GPP3) guidelines, and was funded by Merck Sharp \& Dohme Corp., a subsidiary of Merck \& Co., Inc., Kenilworth, NJ, USA.

\section{Declarations}

Funding This work was supported by Merck Sharp \& Dohme Corp., a subsidiary of Merck \& Co., Inc., Kenilworth, NJ, USA. Medical writ- 
ing support was funded by Merck Sharp \& Dohme Corp., a subsidiary of Merck \& Co., Inc., Kenilworth, NJ, USA. Fees for Open Access were funded by Merck Sharp \& Dohme Corp., a subsidiary of Merck \& Co., Inc., Kenilworth, NJ, USA.

Conflict of interest YT, SM-I, HW, VT, and CB are current or former employees of Merck Sharp \& Dohme Corp., a subsidiary of Merck \& Co., Inc., Kenilworth, NJ, USA (MSD), and may own stock and/or stock options in Merck \& Co., Inc., Kenilworth, NJ, USA. YG is an advisor and speaker for Allergan, MSD, Nabriva, Paratek, and Pfizer, a speaker for Sanofi, and an advisor to Shionogi. SD is an advisor, consultant, and speaker for MSD, an advisory board member for Janssen, and an investigator for Ansun Biopharma, Chimerix, MSD, and Shire/Takeda.

Availability of data and material Merck Sharp \& Dohme Corp., a subsidiary of Merck \& Co., Inc., Kenilworth, NJ, USA (MSD) is committed to providing qualified scientific researchers access to anonymized patient-level data and clinical study reports from the company's clinical trials for the purpose of conducting legitimate scientific research. The company is also obligated to protect the rights and privacy of trial participants and, as such, has a procedure in place for evaluating and fulfilling requests for sharing company clinical trial data with qualified external scientific researchers. The process includes submission of data requests to the MSD data-sharing website available at http://engagezone.msd.com/ds_documentation.php. Data will be made available for request after product approval in the US and EU or after product development is discontinued. There are circumstances that may prevent MSD from sharing the requested data.

Ethics approval This post-hoc analysis is based on data from the phase III clinical trial (NCT02137772), which was conducted in accordance with the principles of the Declaration of Helsinki and Good Clinical Practice guidelines. The institutional review board at each center approved the trial.

Consent This post-hoc analysis is based on data from the phase III clinical trial (NCT02137772). All patients from the trial provided written informed consent.

Author contributions YG participated as a principal investigator, in the study data analysis, and in the writing of the manuscript. SM-I participated in the study analysis plan, performed and reviewed analyses, interpreted data, and critically reviewed the manuscript. YT, HW, and VT participated in the study analysis plan, interpreted data, and critically reviewed the manuscript. CB participated as the clinical director of the study, in the design and conduct of the study, interpreted data, and critically reviewed the manuscript. SD participated as senior/ co-investigator, in the study design and data analysis, and critically reviewed the manuscript.

Open Access This article is licensed under a Creative Commons Attribution-NonCommercial 4.0 International License, which permits any non-commercial use, sharing, adaptation, distribution and reproduction in any medium or format, as long as you give appropriate credit to the original author(s) and the source, provide a link to the Creative Commons licence, and indicate if changes were made. The images or other third party material in this article are included in the article's Creative Commons licence, unless indicated otherwise in a credit line to the material. If material is not included in the article's Creative Commons licence and your intended use is not permitted by statutory regulation or exceeds the permitted use, you will need to obtain permission directly from the copyright holder. To view a copy of this licence, visit http://creativecommons.org/licenses/by-nc/4.0/.

\section{References}

1. Maziarz RT, Hao Y, Guerin A, et al. Economic burden following allogeneic hematopoietic stem cell transplant in patients with diffuse large B-cell lymphoma. Leuk Lymphoma. 2018;59(5):1133-42.

2. Webb BJ, Harrington R, Schwartz J, et al. The clinical and economic impact of cytomegalovirus infection in recipients of hematopoietic stem cell transplantation. Transpl Infect Dis. 2018;20(5):e12961.

3. Schelfhout J, Bonafede M, Cappell K, Cole AL, Manjelievskaia J, Raval AD. Impact of cytomegalovirus complications on resource utilization and costs following hematopoietic stem cell transplant. Curr Med Res Opin. 2020;36(1):33-41.

4. El Haddad L, Ghantoji SS, Park AK, et al. Clinical and economic burden of pre-emptive therapy of cytomegalovirus infection in hospitalized allogeneic hematopoietic cell transplant recipients. J Med Virol. 2020;92(1):86-95.

5. Robin C, Hemery F, Dindorf C, et al. Economic burden of preemptive treatment of CMV infection after allogeneic stem cell transplantation: a retrospective study of 208 consecutive patients. BMC Infect Dis. 2017;17(1):747.

6. Merck Sharp \& Dohme Corp., Whitehouse Station, NJ, USA. PREVYMIS ${ }^{\text {TM }}$ (letermovir) Prescribing Information. 2020. https://www.merck.com/product/usa/pi_circulars/p/prevymis/ prevymis_pi.pdf. Accessed 18 Jan 2021.

7. Marty FM, Ljungman P, Chemaly RF, et al. Letermovir prophylaxis for cytomegalovirus in hematopoietic-cell transplantation. N Engl J Med. 2017;377(25):2433-44.

8. Ljungman P, Schmitt M, Marty FM, et al. A mortality analysis of letermovir prophylaxis for cytomegalovirus (CMV) in CMVseropositive recipients of allogeneic hematopoietic cell transplantation. Clin Infect Dis. 2020;70(8):1525-33.

9. Styczynski J. Who is the patient at risk of CMV recurrence: a review of the current scientific evidence with a focus on hematopoietic cell transplantation. Infect Dis Ther. 2018;7(1):1-16.

10. Schelfhout J, Brown H, House JA, Raval AD. Cytomegalovirus infection and associated hospitalization and costs among individuals undergoing allogeneic hematopoietic stem cell transplant. Curr Med Res Opin. 2020;36(1):43-50.

11. Green ML, Leisenring W, Xie H, et al. Cytomegalovirus viral load and mortality after haemopoietic stem cell transplantation in the era of pre-emptive therapy: a retrospective cohort study. Lancet Haematol. 2016;3(3):e119-27.

12. Alsumali A, Chemaly RF, Graham J, et al. Cost-effectiveness analysis of cytomegalovirus prophylaxis in allogeneic hematopoietic cell transplant recipients from a US payer perspective. J Med Virol. 2020; Online ahead of print.

13. Gajewski JL, McClellan MB, Majhail NS, et al. Payment and care for hematopoietic cell transplantation patients: toward a specialized medical home for complex care patients. Biol Blood Marrow Transpl. 2018;24(1):4-12. 